\title{
Modified Theory of Physical Optics Approach to Diffraction by an Interface between PEMC and Absorbing Half-Planes
}

\author{
Yusuf Z. Umul \\ Department of Electronic and Communication Engineering, Cankaya University, Eskisehir yolu 29. km, Etimesgut 06790, Ankara, Turkey \\ Corresponding author: Yusuf Z. Umul (e-mail: z.umul@cankaya.edu.tr).
}

\begin{abstract}
The scattering of electromagnetic plane waves by an interface, located between perfect electromagnetic conductor and absorbing half-planes, is investigated. The perfect electromagnetic conductor half-plane is divided into perfect electric conductor and perfect magnetic conductor half-screens. The same decomposition is done for the absorbing surface. Then four different geometries are defined according to this approach. The scattered fields by the four sub-problems are obtained with the aid of the modified theory of physical optics. The resultant scattering integrals are combined in a single expression by using key formulas, defined for the perfect electromagnetic conductor and absorbing surfaces. The diffracted and geometric optics fields are obtained by the asymptotic evaluation of the scattering integral for large values of the wave-number. The behaviors of the derived field expressions are analyzed numerically.
\end{abstract}

INDEX TERMS Diffraction theory, high-frequency techniques, modified theory of physical optics, perfect absorber, perfect electromagnetic conductor.

\section{INTRODUCTION}

7 HE method of physical optics (PO) is widely used in electromagnetics for scattering analysis [1,2], wave propagation in waveguides [3], antennas [4] and radar applications $[5,6]$. PO is based on the integration of an induced surface current over the illuminated part of the scatterer. However, the technique has an important defect. The edge diffracted waves, evaluated from the PO integral, are not correct [7]. The reason of this problem is due to the evaluation of the surface current. The incident and reflected geometric optics $(\mathrm{GO})$ waves are used for the evaluation of the current. The two GO fields have the same angle according to the unit normal vector of the surface. However, the angle between the diffracted wave and incident GO field are not same according to this normal vector. For this reason, the current should be evaluated with respect to a variable unit vector instead of the static one. By using this proposal, we proposed a method, named as the modified theory of physical optics (MTPO), that was leading to the exact edge diffracted fields for the perfect electric conductor (PEC) and perfect magnetic conductor (PMC) half-planes in 2004 [8]. The application of MTPO to the diffraction problem of waves by an impedance halfplane yielded diffracted wave expressions which were in harmony with the exact solution of the problem [9]. Later we extended the method for junction type problems by defining the concept of initial fields [10]. With the aid of this concept, we proposed a new method, which is named as the method of transition boundary (MTB). It was proposed for the evaluation of the diffracted waves for two dimensional problems [11]. In MTB, the diffracted fields were obtained with the aids of factorization and principle of reciprocity. The process of factorization was carried out in the real space as opposed to the methods of Wiener-Hopf [12] and plane wave spectrum integral [13]. We integrated the method of factorization to MTPO in order to extend its applicability to the class of problems for which the rigorous solutions could be found [14].

The aim of this paper is to investigate the scattering problem of electromagnetic plane waves by a planar interface between perfect electromagnetic conductor (PEMC) and perfect absorber (PA) half-planes. To our knowledge, this problem has not been investigated yet. Such geometry is important for the determination of the discontinuity effects between two surfaces that are defined in terms of different boundary conditions. For example, the aircrafts with low radar cross section will give rise to the diffracted fields if there are any discontinuities between the absorbing and reflecting parts of the surface. The absorbing superficies of the aircraft can be modeled by the boundary condition of a perfect absorber. We will use the method of MTPO for the evaluation of the diffracted waves by the planar junction. It was shown in previous studies that the scattered fields by PEMC or PA surfaces could be expressed as a combination of the scattered waves by PEC and PMC surfaces with the same geometries $[14,15]$. With the aid of this information, 
we will decompose the geometry of the scenario to subproblems. Then the solutions of the separate junction problems will be combined in order to obtain the diffracted waves, related with the main problem. The expressions of the total, total GO and diffracted fields will be derived by the asymptotic evaluation of the MTPO scattering integral for large values of the wave-number $k$. Some numerical results will be given for the behaviors of the obtained field expressions.

A time factor of $\exp (j \omega t)$ is assumed and suppressed throughout the paper. $\omega$ is the angular frequency.

\section{DEFINITION OF THE PROBLEM}

The geometry of the problem is given in Fig. 1. The PEMC and PA half-planes are located at $x>0, y=0, z \in(-\infty, \infty)$ and $x<0, y=0, z \in(-\infty, \infty)$ respectively. $P$ is the observation point. The incident electric field is expressed by

$$
\vec{E}_{i}=E_{0} e^{j k\left(x \cos \phi_{0}+y \sin \phi_{0}\right)} \vec{e}_{z}
$$

where $E_{0}$ is the complex amplitude and $\phi_{0}$ angle of incidence. The Cartesian and polar coordinates are shown by $(x, y, z)$ and $(\rho, \phi, z)$ respectively.

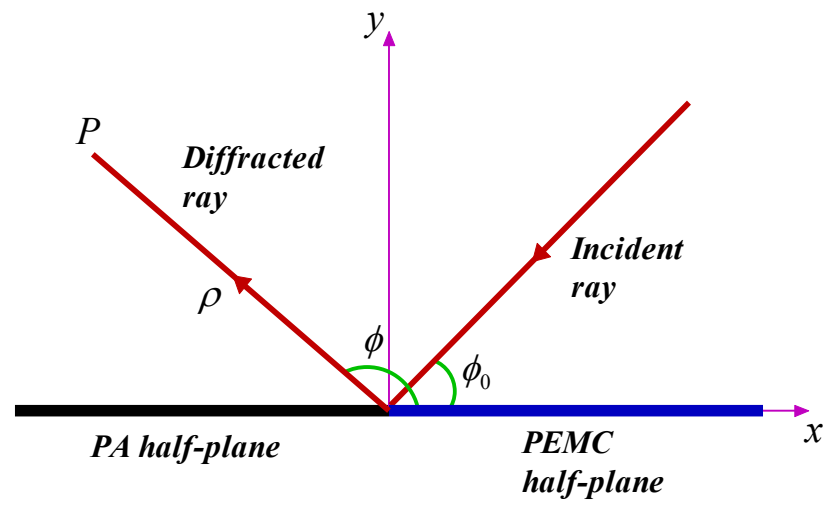

FIGURE 1. The diffraction geometry of waves by a planar junction between PEMC and PA half-planes.

A PEMC surface is a combination of the PEC and PMC surfaces. The boundary condition on such a surface is given by

$$
\vec{n} \times\left(\vec{H}_{T}+M \vec{E}_{T}\right)_{S}=0
$$

for $\vec{n}$ is the unit normal vector of the surface [17]. $M$ is a parameter. The PEMC becomes PEC or PMC when $M$ goes to infinity or zero respectively. The subscript $T$ means total. The sum of the incident and reflected fields can be written as

$$
\vec{E}_{T}=\frac{E_{h}+Z_{0}^{2} M^{2} E_{s}}{1+Z_{0}^{2} M^{2}} \vec{e}_{z}
$$

and

$$
\vec{H}_{T}=-\frac{M\left(E_{h}-E_{s}\right)}{1+Z_{0}^{2} M^{2}} \vec{e}_{z}
$$

for a whole PEMC surface, located at $y=0$ and illuminated by the incident field, given in Eq. (1) [16]. $Z_{0}$ is the impedance of free space. $E_{s}$ and $E_{h}$ are the total electric fields for PEC and PMC whole-planes respectively. Here, $H_{T}$ is the crosspolarized field, excited by the PEMC surface. The total field is the summation of the incident and reflected waves in this case. Eqs. (3) and (4) provide a unique method for the evaluation of the scattered waves by an obstacle with PEMC boundary condition. First of all, the scattered fields will be evaluated for the same obstacle with PEC and PMC boundary conditions. Then, the derived field expressions will be combined by using Eqs. (3) and (4) in order to obtain the scattered electric and magnetic fields.

A PA layer absorbs all of the electromagnetic energy that impinges on its surface. Such materials can be realized by using metasurfaces $[18,19]$. The total electric field can be expressed by

$$
\vec{E}_{T}=\frac{E_{s}+E_{h}}{2} \vec{e}_{z}
$$

for a PA whole-plane, located at $y=0$. The surface is illuminated by the electric field, in Eq. (1) [20]. The scattered waves by a PA obstacle can be evaluated with the aid of Eq. (5) as in a PEMC surface.

\section{DECOMPOSITION OF THE PROBLEM}

In this section, we will decompose the geometry of the problem, given in Fig. 1, by using the MTPO. First of all, we will decompose the geometry into two parts as shown in Figs. 2.a and 2.b. (a)

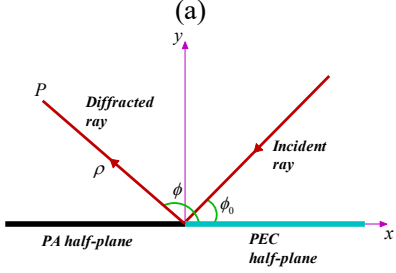

(b)

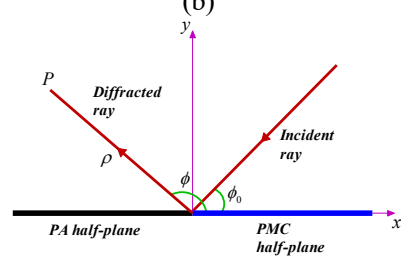

FIGURE 2. The diffraction geometry a) by a planar junction between PEC and PA half-planes, b) by a planar junction between PMC and PA halfplanes.

We assume that the $z$ components of the diffracted electric fields are shown by $E_{d s}$ and $E_{d h}$ for the cases in Figs. 2.a and 2.b respectively. The total diffracted electric and magnetic fields can be written as

$$
E_{d}=\frac{E_{d h}+Z_{0}^{2} M^{2} E_{d s}}{1+Z_{0}^{2} M^{2}}
$$

and 


$$
H_{d}=-\frac{M\left(E_{d h}-E_{d s}\right)}{1+Z_{0}^{2} M^{2}}
$$

for the problem under consideration. These equations are written according to Eqs. (3) and (4). Thus, $E_{d s}$ and $E_{d h}$ should be evaluated in order to find the solution of the problem. With this aim, Figs. 3.a and 3.b are taken into account. This figure is the decomposition of the problem, given in Fig. 2.a. The PA half-plane is written in terms of the PEC and PMC half-screens. The diffracted fields are expressed by $E_{d s s}$ and $E_{d s h}$ for the geometries, in Figs. 3.a and 3.b respectively. The diffracted electric field $E_{d s}$ can be evaluated by the equation of

$$
E_{d s}=\frac{E_{d s s}+E_{d s h}}{2}
$$

according to Eq. (5). Since there will not be any discontinuity at the junction of two PEC half-planes, $E_{d s s}$ is equal to zero. $E_{d s}$ becomes

$$
E_{d s}=\frac{E_{d s h}}{2}
$$

in this case.

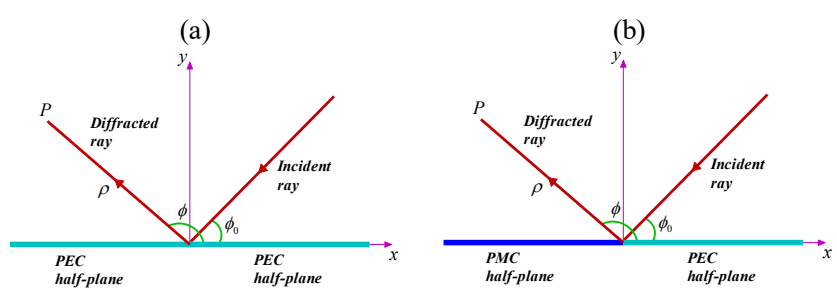

FIGURE 3. The diffraction geometry a) by a planar junction between PEC and PEC half-planes, b) by a planar junction between PEC and PMC halfplanes. (a)

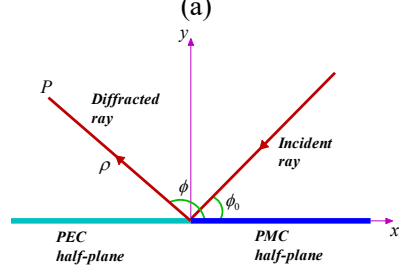

(b)

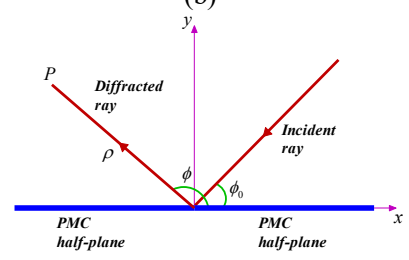

FIGURE 4. The diffraction geometry a) by a planar junction between PMC and PEC half-planes, b) by a planar junction between PMC and PMC halfplanes.

A similar decomposition can be applied to the geometry, in Fig. 2.b. Figure 4.a and $b$ are taken into consideration. The diffracted fields are $E_{d h s}$ and $E_{d h h}$ for this situation. $E_{d h}$ can be evaluated from the equation

$$
E_{d h}=\frac{E_{d h s}+E_{d h h}}{2} .
$$

There will not be any diffraction by a whole PMC plane. For this reason, $E_{d h h}$ is zero. $E_{d h}$ becomes

$$
E_{d h}=\frac{E_{d h s}}{2}
$$

in this case.

The total diffracted electric and magnetic fields read

$$
E_{d}=\frac{E_{d h s}+Z_{0}^{2} M^{2} E_{d s h}}{2\left(1+Z_{0}^{2} M^{2}\right)}
$$

and

$$
H_{d}=-\frac{M\left(E_{d h s}-E_{d s h}\right)}{2\left(1+Z_{0}^{2} M^{2}\right)}
$$

respectively when Eqs. (9) and (11) are considered.

\section{CONSTRUCTION OF THE MTPO SCATTERING INTEGRALS}

The total electric field can be written as

$$
\vec{E}_{T}=\vec{E}_{i}+\vec{E}_{S}
$$

when the incident electric field $E_{i}$ interacts with an obstacle on its path of propagation. $E_{S}$ is the scattered field, which has the expression of

$$
\vec{E}_{S}=\frac{j k}{2 \pi} \iint_{A} \vec{f}\left(A^{\prime}\right) \frac{e^{-j k R_{1}}}{R_{1}} d A^{\prime}
$$

in terms of the MTPO integral. $A$ is the surface of the scatterer. $R_{1}$ is the distance between the integration and observation points. $f$ is a function to be determined. In this study, we will use the factorization method for this aim [14]. The MTPO integral can be represented as

$$
\vec{E}_{S}=\frac{k e^{j \frac{\pi}{4}}}{\sqrt{2 \pi}} \int_{C} \vec{f}\left(l^{\prime}\right) \frac{e^{-j k R}}{\sqrt{k R}} d l^{\prime}
$$

for two dimensional problems. In some cases, it is more appropriate to use the initial waves instead of the incident field [11]. Initial waves are the fields that occur in space when the scatterer that causes discontinuity is excluded.

First of all, the geometry, in Fig. 3.a, is taken into account. Since the scatterer is a PEC whole-plane in this case, there will not be any diffracted fields. The electric field can be written by

$$
\vec{E}_{T 1}=\vec{E}_{i}-\vec{E}_{r}
$$

as a sum of the incident and reflected waves. The reflected electric field has the representation of

$$
\vec{E}_{r}=E_{0} e^{j k\left(x \cos \phi_{0}-y \sin \phi_{0}\right)} \vec{e}_{z} .
$$

As the second problem, we consider the scatterer, in Fig. 3.b. In this situation, the initial fields will be determined. With this aim, the PEC half-plane is excluded from space and only a PMC whole-plane exists. The initial fields read 


$$
\vec{E}_{i n 2}=\vec{E}_{i}+\vec{E}_{r}
$$

for the case under consideration. The total GO field can be written as

$$
\vec{E}_{T G O 2}=\vec{E}_{i}-\vec{E}_{r} U\left(-\xi_{+}\right)+\vec{E}_{r} U\left(\xi_{+}\right)
$$

directly from the geometry, in Fig. 3.b. $U(x)$ is the unit step function, which is equal to 1 for $x>0$ and 0 otherwise. The detour parameter $\xi_{ \pm}$can be defined by

$$
\xi_{ \pm}=-\sqrt{2 k \rho} \cos \frac{\phi \pm \phi_{0}}{2} .
$$

The scattered GO field is found to be

$$
\vec{E}_{S G O 2}=-2 \vec{E}_{r} U\left(-\xi_{+}\right)
$$

from the difference of the initial and total GO waves [11]. The total field can be given by

$$
\vec{E}_{T 2}=\vec{E}_{i n 2}+\vec{E}_{S 2}
$$

for the scattered field is introduced as

$$
\vec{E}_{S 2}=\vec{e}_{z} \frac{k E_{0} e^{j \frac{\pi}{4}}}{\sqrt{2 \pi}} \int_{0}^{\infty} f(\beta) e^{j k x^{\prime} \cos \phi_{0}} \frac{e^{-j k R}}{\sqrt{k R}} d x^{\prime}
$$

in terms of the MTPO integral [14]. $\beta$ is the angle of scattering and is a function of $x^{\prime}$. The scattered GO wave, in Eq. (22), will be used in the determination of the function $f$. The relation of

$$
f\left(\phi_{0}\right)=-2 \sin \phi_{0}
$$

can be written at $\beta=\phi_{0}$, which is the location of the reflection boundary [14]. Eq. (24) can be rewritten as

$$
f\left(\phi_{0}\right)=-4 \sin \frac{\phi_{0}}{2} \cos \frac{\phi_{0}}{2} \text {. }
$$

The function $f$ can be expressed by

$$
f(\beta)=-4 \cos \frac{\beta}{2} \sin \frac{\phi_{0}}{2}
$$

in order to satisfy the boundary conditions. This choice will be clear when the edge diffracted field is evaluated. Thus the MTPO integral can be written as

$$
\vec{E}_{S 2}=-\vec{e}_{z} \frac{4 k E_{0} e^{j \frac{\pi}{4}}}{\sqrt{2 \pi}} \int_{0}^{\infty} \cos \frac{\beta}{2} \sin \frac{\phi_{0}}{2} e^{j k x^{\prime} \cos \phi_{0}} \frac{e^{-j k R}}{\sqrt{k R}} d x^{\prime}
$$

for the geometry, in Fig. 3.b. The total field can be evaluated as

$$
\vec{E}_{T S}=\vec{E}_{i}+\frac{\vec{E}_{S 2}}{2}
$$

for the scatterer in Fig. 2.a. Eq. (5) is used in the evaluation of Eq. (29).

The geometry, in Fig. 4.a, is taken into consideration. First of all, the PMC half-plane, at the right-hand side, will be excluded and the initial fields will be determined for a whole PEC plane. These fields can be written as

$$
\vec{E}_{i n 3}=\vec{E}_{i}-\vec{E}_{r} .
$$

The total fields have the expression of

$$
\vec{E}_{T G O 3}=\vec{E}_{i}+\vec{E}_{r} U\left(-\xi_{+}\right)-\vec{E}_{r} U\left(\xi_{+}\right)
$$

according to the geometry, in Fig. 4.a. The scattered GO wave is found to be

$$
\vec{E}_{S G O 3}=2 \vec{E}_{r} U\left(-\xi_{+}\right)
$$

in this case. The MTPO integral can be expressed as

$$
\vec{E}_{S 3}=\vec{e}_{z} \frac{k E_{0} e^{j \frac{\pi}{4}}}{\sqrt{2 \pi}} \int_{0}^{\infty} f(\beta) e^{j k x^{\prime} \cos \phi_{0}} \frac{e^{-j k R}}{\sqrt{k R}} d x^{\prime}
$$

and the function $f$ will be determined according to Eq. (32). The relation of

$$
f\left(\phi_{0}\right)=2 \sin \phi_{0}
$$

can be written. The function can be chosen as

$$
f(\beta)=4 \sin \frac{\beta}{2} \cos \frac{\phi_{0}}{2}
$$

by taking into account the boundary conditions on the halfplanes. Then the MTPO scattering integral is given by

$$
\vec{E}_{S 3}=\vec{e}_{z} \frac{4 k E_{0} e^{j \frac{\pi}{4}}}{\sqrt{2 \pi}} \int_{0}^{\infty} \sin \frac{\beta}{2} \cos \frac{\phi_{0}}{2} e^{j k x^{\prime} \cos \phi_{0}} \frac{e^{-j k R}}{\sqrt{k R}} d x^{\prime}
$$

when Eq. (35) is used in Eq. (33). At this point, it is important to note that $R$ has the expression of

$$
R=\sqrt{\left(x-x^{\prime}\right)^{2}+y^{2}}
$$

in the two dimensional space. The total electric field can be given by the equation

$$
\vec{E}_{T 3}=\vec{E}_{i n 3}+\vec{E}_{S 3}
$$

for the case, under consideration.

The last scatterer is given in Fig. 4.b. Since it shows a whole PMC plane, the diffraction process does not occur. The total electric field can be written by

$$
\vec{E}_{T 4}=\vec{E}_{i}+\vec{E}_{r}
$$

in this situation. The total electric field can be represented as 


$$
\vec{E}_{T h}=\vec{E}_{i}+\frac{\vec{E}_{S 3}}{2}
$$

for the geometry, in Fig. 2.b.

The total electric and magnetic fields can be expressed by

$$
\vec{E}_{T}=\frac{\vec{E}_{T h}+Z_{0}^{2} M^{2} \vec{E}_{T s}}{1+Z_{0}^{2} M^{2}}
$$

and

$$
\vec{H}_{T}=-\frac{M\left(\vec{E}_{T h}-\vec{E}_{T s}\right)}{1+Z_{0}^{2} M^{2}}
$$

from Eqs. (3) and (4) respectively.

\section{ASYMPTOTIC EVALUATION OF THE MTPO}

\section{INTEGRALS}

The integrals, in Eqs. (28) and (36), are taken into consideration. They will be asymptotically evaluated for large values of the wave-number. Only the diffracted waves will be obtained by using the edge point method. The phase functions of the integrals, in Eqs. (28) and (36), are the same and equal to

$$
g\left(x^{\prime}\right)=x^{\prime} \cos \phi_{0}-R .
$$

The first derivative of Eq. (43) reads

$$
\frac{d g}{d x^{\prime}}=\cos \phi_{0}-\cos \beta
$$

according to the geometry, in Fig. 5.

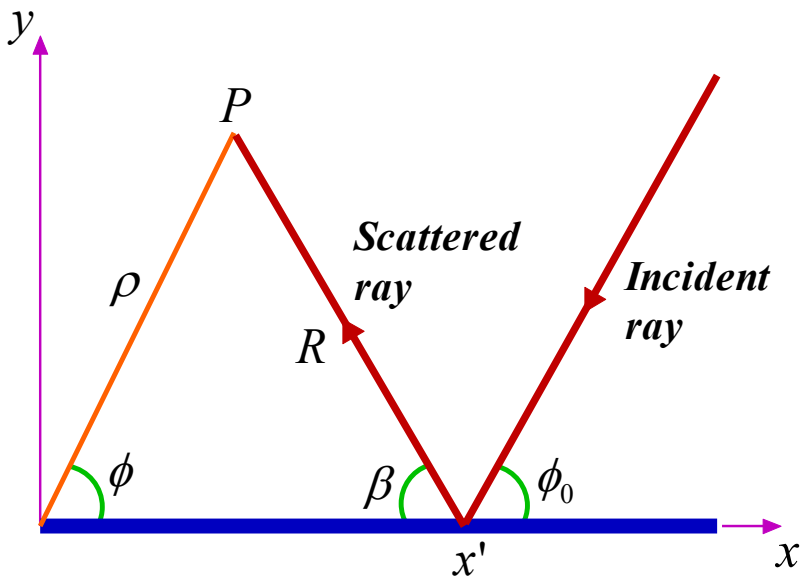

FIGURE 5. The scattering geometry by a half-plane.

The edge point evaluation of a scattering integral can be given by the formula

$$
\int_{x_{e}}^{\infty} h(x) e^{j k g(x)} d x \approx-\frac{1}{j k} \frac{f\left(x_{e}\right)}{g^{\prime}\left(x_{e}\right)} e^{j k g\left(x_{e}\right)}
$$

where $h(x)$ is the amplitude function [21]. $x_{e}$ shows the edge point. First of all, Eq. (28) is considered. The edge point evaluation yields

$$
\vec{E}_{d s h}=\frac{4 E_{0} e^{-j \frac{\pi}{4}}}{\sqrt{2 \pi}} \frac{\sin \frac{\phi}{2} \sin \frac{\phi_{0}}{2}}{\cos \phi+\cos \phi_{0}} \frac{e^{-j k \rho}}{\sqrt{k \rho}} \vec{e}_{z}
$$

for edge point is equal to $0 . R$ and $\beta$ becomes $\rho$ and $\pi-\phi$ at the edge point of the integral respectively. The asymptotic evaluation of the integral, in Eq. (36), gives

$$
\vec{E}_{d h s}=-\frac{4 E_{0} e^{-j \frac{\pi}{4}}}{\sqrt{2 \pi}} \frac{\cos \frac{\phi}{2} \cos \frac{\phi_{0}}{2}}{\cos \phi+\cos \phi_{0}} \frac{e^{-j k \rho}}{\sqrt{k \rho}} \vec{e}_{z} .
$$

$E_{d s}$ and $E_{d h}$ can be expressed by

$$
\vec{E}_{d s}=\frac{E_{0} e^{-j \frac{\pi}{4}}}{\sqrt{2 \pi}} \frac{2 \sin \frac{\phi}{2} \sin \frac{\phi_{0}}{2}}{\cos \phi+\cos \phi_{0}} \frac{e^{-j k \rho}}{\sqrt{k \rho}} \vec{e}_{z}
$$

and

$$
\vec{E}_{d h}=-\frac{E_{0} e^{-j \frac{\pi}{4}}}{\sqrt{2 \pi}} \frac{2 \cos \frac{\phi}{2} \cos \frac{\phi_{0}}{2}}{\cos \phi+\cos \phi_{0}} \frac{e^{-j k \rho}}{\sqrt{k \rho}} \vec{e}_{z}
$$

according to Eqs. (9) and (11) respectively. The total diffracted electric and magnetic fields can be written as

$$
E_{d}=-2 \frac{E_{0} e^{-j \frac{\pi}{4}}}{\sqrt{2 \pi}} \frac{\cos \frac{\phi}{2} \cos \frac{\phi_{0}}{2}-Z_{0}^{2} M^{2} \sin \frac{\phi}{2} \sin \frac{\phi_{0}}{2}}{\left(1+Z_{0}^{2} M^{2}\right)\left(\cos \phi+\cos \phi_{0}\right)} \frac{e^{-j k \rho}}{\sqrt{k \rho}}
$$

and

$$
H_{d}=2 \frac{E_{0} e^{-j \frac{\pi}{4}}}{\sqrt{2 \pi}} \frac{M\left(\cos \frac{\phi}{2} \cos \frac{\phi_{0}}{2}+\sin \frac{\phi}{2} \sin \frac{\phi_{0}}{2}\right)}{\left(1+Z_{0}^{2} M^{2}\right)\left(\cos \phi+\cos \phi_{0}\right)} \frac{e^{-j k \rho}}{\sqrt{k \rho}}
$$

when Eqs. (12) and (13) are taken into account. Note that only $z$ components of the diffracted fields are considered. These field expressions tend to infinity at the reflection $\left(\pi-\phi_{0}\right)$ and shadow $\left(\pi+\phi_{0}\right)$ boundaries. The uniform fields $\mathrm{cn}$ be expressed by

$$
E_{d}=E_{0} \frac{p_{h}+Z_{0}^{2} M^{2} p_{s}}{1+Z_{0}^{2} M^{2}}
$$

and

$$
H_{d}=E_{0} \frac{M\left(p_{s}-p_{h}\right)}{1+Z_{0}^{2} M^{2}}
$$

for $p_{s}$ and $p_{h}$ can be defined as 


$$
\begin{aligned}
p_{s} & =e^{j k \rho \cos \left(\phi-\phi_{0}\right)} \operatorname{sign}\left(\xi_{-}\right) F\left[\left|\xi_{-}\right|\right] \\
& -e^{j k \rho \cos \left(\phi+\phi_{0}\right)} \operatorname{sign}\left(\xi_{+}\right) F\left[\left|\xi_{+}\right|\right]
\end{aligned}
$$

and

$$
\begin{aligned}
p_{h} & =e^{j k \rho \cos \left(\phi-\phi_{0}\right)} \operatorname{sign}\left(\xi_{-}\right) F\left[\left|\xi_{-}\right|\right] \\
& +e^{j k \rho \cos \left(\phi+\phi_{0}\right)} \operatorname{sign}\left(\xi_{+}\right) F\left[\left|\xi_{+}\right|\right]
\end{aligned}
$$

respectively. $\operatorname{sign}(x)$ is the signum function, which is equal to 1 for $x>0$ and -1 for $x<0 . F[x]$ is the Fresnel function, which can be introduced by the integral

$$
F[x]=\frac{e^{j \frac{\pi}{4}}}{\sqrt{\pi}} \int_{x}^{\infty} e^{-j v^{2}} d v .
$$

The total electric and magnetic fields can be written as

$$
E_{T}=E_{T G O}+E_{d}
$$

and

$$
H_{T}=H_{T G O}+H_{d}
$$

where $E_{T G O}$ and $H_{T G O}$ can be expressed by

$$
E_{T G O}=E_{i}+\frac{1-Z_{0}^{2} M^{2}}{1+Z_{0}^{2} M^{2}} E_{r} U\left(-\xi_{+}\right)
$$

and

$$
H_{T G O}=-\frac{2 M}{1+Z_{0}^{2} M^{2}} E_{r} U\left(-\xi_{+}\right)
$$

respectively [16]. Note that the $z$ component of the magnetic field is a cross-polarized wave. For this reason, there is not any incident field in Eq. (60). $p_{s}$ and $p_{h}$, in Eqs. (54) and (55), are the diffracted waves by PEC and PMC half-planes.

\section{NUMERICAL RESULTS}

In this section we will analyze numerically the behavior of the total electric and magnetic fields with their components numerically. The distance of observation $(\rho)$ is taken as $6 \lambda . \lambda$ is the wavelength. The angle of observation $(\phi)$ varies between $0^{\circ}$ and $180^{\circ}$. The angle of incidence is equal to $60^{\circ}$. $Z_{0}$ has the value of $377 \Omega . M$ is equal to 3 .

Figure 6 shows the variation of the total GO and diffracted electric fields versus the observation angle. The total GO field shows interference for observation angle values, smaller than $120^{\circ}$. In this region, the reflected wave from the PEMC surface and the incident electric field interfere with each other. After $120^{\circ}$, the reflected GO wave goes to zero and there is not any reflected field from the PA half-plane. For this reason, only the incident wave is observed. The diffracted wave takes its maximum value at the reflection boundary, which is located at $120^{\circ}$. In fact it has also a phase shift of $180^{\circ}$ at this location. Note that the total GO field has also a discontinuity at the reflection boundary.

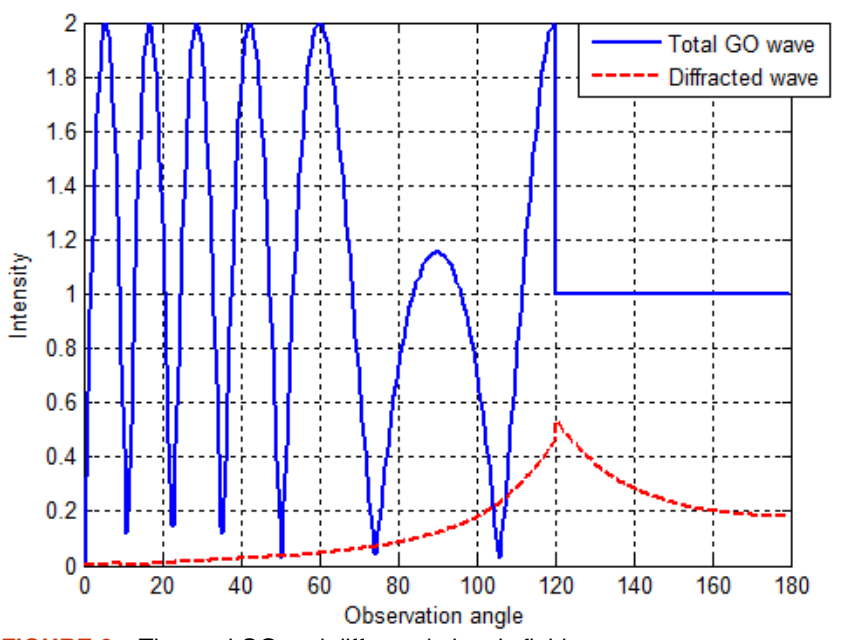

FIGURE 6. The total GO and diffracted electric fields.

In Fig. 7, the total electric field's $z$ component is plotted. It is the summation of the total GO and diffracted fields, shown in Fig .6. It can be seen from Fig. 7 that the total field is continuous everywhere in space. After $120^{\circ}$, the interference of the incident wave and diffracted field is observed.

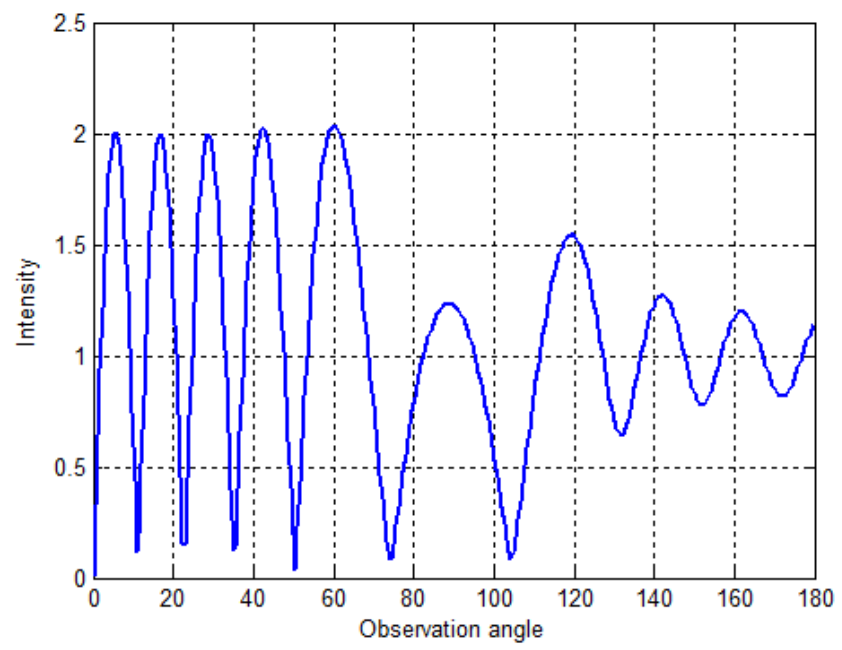

FIGURE 7. The total electric field.

Figure 8 depicts the variations of the magnetic field's total $\mathrm{GO}$ and diffracted components. Also the $z$ component is taken into account in this case. Since the $z$ component of the magnetic field is cross-polarized, an incident field does not exist for this polarization. Thus only the reflected magnetic field from the PEMC half-plane is observed in the figure. After $120^{\circ}$, this field suddenly goes to zero and creates a discontinuity. There is not any reflected magnetic field from the PA half-plane. The magnetic field takes it maximum value at this location. The total magnetic field, which is the sum of the total GO and diffracted waves, is given in Fig. 9. For observation angle values, smaller that $120^{\circ}$, the 
interference of the total GO and diffracted field is observed. After the reflection boundary, only the diffracted magnetic field exists. As can be seen from the figure, the total magnetic field is continuous.

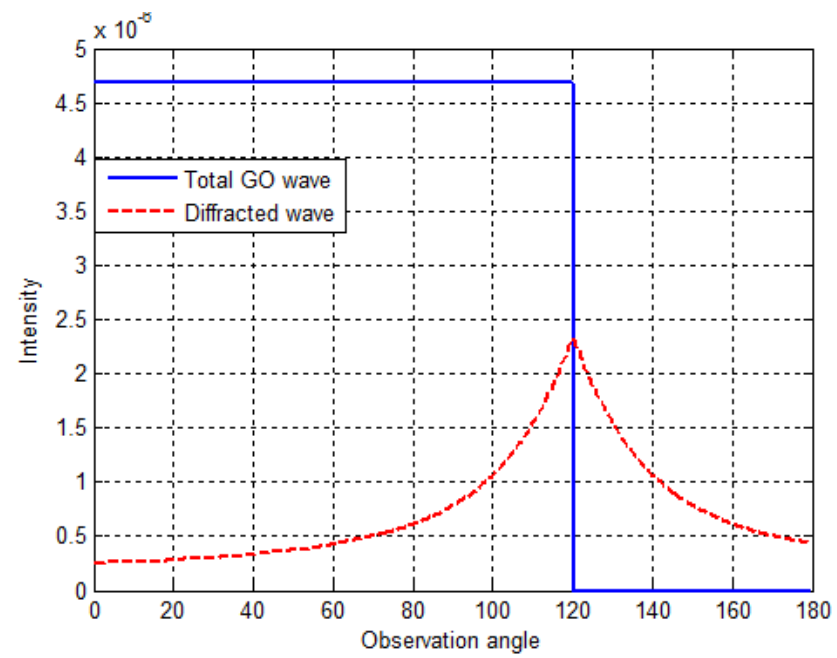

FIGURE 8. The total GO and diffracted magnetic fields.

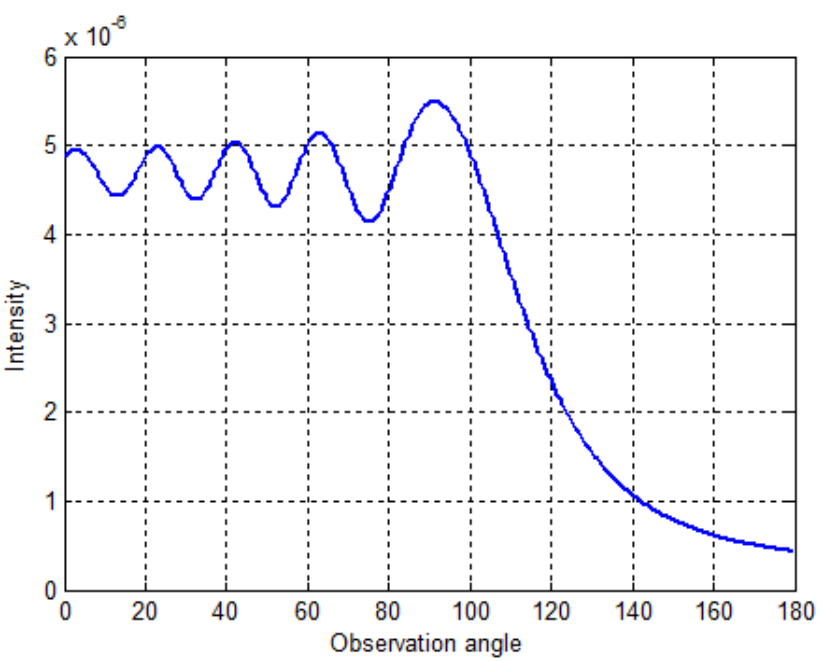

FIGURE 9. The total magnetic field.

\section{CONCLUSION}

In this paper, we investigated the diffraction problem of electromagnetic waves by a planar interface between PEMC and PA half-screens. The method of MTPO is used for the determination of the scattered fields. Although the asymptotic evaluation of the MTPO integral gives both of the scattered GO and diffracted waves, we only evaluated the diffraction effect by the technique of edge point. The GO waves can be easily determined by using the geometry and the related reflection coefficient of a PEMC surface. The total field is obtained by the summation of the total GO and diffracted fields. The behaviors of the total electric and magnetic fields with their components studied numerically. It is important to note that the diffracted electric and magnetic fields, given in Eqs. (50) and (51), are the high-frequency asymptotic expressions, which are valid for the conditions of $k \gg>1$ and $\lambda \rightarrow 0$. They are obtained from the edge point evaluation of the MTPO integrals as shown in Section V. The MTPO integrals, obtained in Section IV, represent the exact solution of the problem under consideration $[8,14,22]$.

\section{REFERENCES}

[1] N. S. Tezel, "Electromagnetic scattering by anisotropic inhomogeneous impedance cylinder of arbitrary shape using physical optics," IEEE Geosci. Remote Sens. Lett., vol. 5, pp. 663-667, Oct. 2008.

[2] Y. Z. Umul, "Physical optics scattering of waves by a half-plane with different face impedances," IEEE Antennas Wirel. Propag. Lett., vol. 10, pp. 21-24, Jan. 2011.

[3] R. Cicchetti and A. Faraone, "Analysis of open-ended circular waveguides using physical optics and incomplete Hankel functions formulation," IEEE Trans. Antennas Propag., vol. 55, pp. 18871892, Jun. 2007.

[4] A. Boag and C. Letrou, "Multilevel fast physical optics algorithm for radiation from non-planar apertures," IEEE Trans. Antennas Propag., vol. 53, pp. 2064-2072, Jun. 2005.

[5] S. Adachi, A. Oashi and T. Uno, "Iterative radar target imaging based on modified extended physical optics method," IEEE Trans. Antennas Propag., vol. 38, pp. 847-852, Jun. 1990.

[6] F. S. de Adana, I. G. Diego, O. G. Blanco, P. Lozano and M. F. Catedra, "Method based on physical optics for the computation of the radar cross section including diffraction and double effects of metallic and absorbing bodies modeled with parametric surfaces," IEEE Trans. Antennas Propag., vol. 52, pp. 3295-3303, Dec. 2004.

[7] W. L. Stutzman and G. A. Thiele, Antenna Theory and Design. $3^{\text {rd }}$ ed., Hoboken, NJ, USA: Wiley, 2013, pp. 750-769.

[8] Y. Z. Umul, "Modified theory of physical optics," Opt. Express, vol. 12, pp. 4959-4972, Oct. 2004.

[9] Y. Z. Umul, "Modified theory of physical optics solution of impedance half plane problem," IEEE Trans. Antennas Propag, vol. 54, pp. 2048-2053, Jul. 2006.

[10] Y. Z. Umul, "Integral theory of diffraction for material junctions," Optik - Int. J. Light electron Opt., vol. 130, pp. 1124-1138, Feb. 2017.

[11] Y. Z. Umul, "The method of transition boundary for the solution of diffraction problems," Opt. Quantum Electron., vol. 51, article no. 181, Jun. 2019.

[12] E. T. Copson, "On an integral equation arising in the theory of diffraction," Q. J. Math., vol. 17, pp. 19-34, 1946.

[13] P. C. Clemmow, "A method for the exact solution of a class of twodimensional diffraction problems," Proc. R. Soc. Lond. A, vol. 205 , pp. 286-308, Feb. 1951

[14] Y. Z. Umul, "The factorization process in the modified theory of physical optics," Optik - Int. J. Light electron Opt., vol. 205, article no. 164249 , Mar. 2020

[15] Y. Z. Umul, "Diffraction of electromagnetic waves by a wedge with perfectly absorbing and impedance faces," Optik - Int. J. Light electron Opt., vol. 174, pp. 416-424, Dec. 2018.

[16] Y. Z. Umul, "Scattering of electromagnetic waves by a perfect electromagnetic conductor half-screen," Optik - Int. J. Light electron Opt., vol. 181, pp. 383-388, Mar. 2019.

[17] I. V. Lindell and A. H. Sihvola, "Perfect electromagnetic conductor," J. Electromagn. Waves Appl., vol. 19, pp. 861-869, 2005.

[18] R. Alee, M. Albooyeh and C. Rockstuhl, "Theory of metasurface based perfect absorbers," J. Phys. D: Appl. Phys., vol. 50, article no. 503002, Nov. 2017.

[19] T. Badloe, J. Mun and J. Rho, "Metasurface-based absorption and reflection control: perfect absorbers and reflectors," J. Nanomater., vol. 2017, article no. 2361042, 2017

[20] Y. Z. Umul, "Diffraction of plane waves by a black wedge," Opt. Laser Technol., vol. 42, pp. 32-36, Feb. 2010.

[21] Y. Z. Umul, "MTPO based potential function of the boundary diffraction wave theory," Opt. Laser Technol., vol. 40, pp. 769-774, Sep. 2008

[22] Y. Z. Umul, "Three dimensional modified theory of physical optics," Otik - Int. J. Light Electron Opt., vol. 127, pp. 819-824. 